\title{
Distribuição de Leptasthenura setaria (Temminck, 1824) (Aves: Furnariidae) no Estado de São Paulo
}

\author{
Alexsander Zamorano Antunes ${ }^{1,7}$, Herculano Alvarenga ${ }^{2}$, Luís Fábio Silveira ${ }^{3}$, Marilda Rapp de Eston ${ }^{4}$ \\ Gisela Vianna Menezes ${ }^{5}$ \& Antônio Silveira Ribeiro dos Santos ${ }^{6}$ \\ Biota Neotropica $v 7(n 1)$ \\ http://www.biotaneotropica.org.br/v7n1/pt/abstract?short-communication+bn00607012007 \\ Recebido em 04/10/06 \\ Versão reformulada recebida em 16/11/06 \\ Publicado em 01/01/07 \\ ${ }^{1}$ Seção de Animais Silvestres, Divisão de Dasonomia Instituto Florestal, Rua do Horto, 931, \\ CP 1322, CEP 01059-970, São Paulo, SP, Brasil \\ ${ }^{2}$ Museu de História Natural de Taubaté, Rua Juvenal Dias de Carvalho, s/n ${ }^{\circ}$ Taubaté, SP, Brasil, \\ e-mail: halvarenga@uol.com.br \\ ${ }^{3}$ Departamento de Zoologia, Instituto de Biociências, Universidade de São Paulo, \\ Rua do Matão, Travessa 14, n. 101, CEP 05508-900, São Paulo, SP, Brasil, \\ e-mail: lfsilvei@usp.br \\ ${ }^{4}$ Instituto Florestal, CP 1322, CEP 01059-970, São Paulo, SP, Brasil, \\ e-mail: marildarapp@iflorestal.sp.gov.br \\ ${ }^{5}$ Instituto Florestal, CP 1322, CEP 01059-970, São Paulo, SP, Brasil, e-mail: gismenezes@ig.com.br \\ ${ }^{6}$ Programa Ambiental A Última Arca de Noé, Avenida Jamaris, 428, apto 142, \\ CEP 04078-001, São Paulo, SP, Brasil, e-mail: arca@aultimaarcadenoe.com \\ ${ }^{7}$ Autor para correspondência: Alexsander Zamorano Antunes, e-mail: alexsanderantunes@ig.com.br
}

\section{Abstract}

Antunes, A.Z., Alvarenga, H., Silveira, F.L., Eston, M.R., Menezes, G.V. \& Santos, S.R. Leptasthenura setaria (Temminck, 1824) (Aves: Furnariidae) in the state of São Paulo, southeastern Brazil. Biota Neotrop. Jan/Apr 2007 vol. 7, no. 1 http://www.biotaneotropica.org.br/v7n1/pt/abstract?short-communication+bn00607012007 ISSN 1676-0603.

The Araucaria Tit-Spinetail Leptasthenura setaria is found exclusively in Parana-Pine Araucaria angustifolia, being classified as near-threatened by IUCN. We present seven new localities to Araucaria Tit-Spinetail from São Paulo, including the first state specimens. This species was recorded in native or planted Parana-Pine, in five reserves.

Keywords: Araucaria angustifolia, Araucaria forest, Araucaria Tit-Spinetail, conservation.

\section{Resumo}

Antunes, A.Z., Alvarenga, H., Silveira, F.L., Eston, M.R., Menezes, G.V. \& Santos, S.R. Distribuição de Leptasthenura setaria (Temminck, 1824) (Aves: Furnariidae) no Estado de São Paulo. Biota Neotrop. Jan/Apr 2007 vol. 7, no. 1 http://www.biotaneotropica.org.br/v7n1/pt/abstract?short-communication+bn00607012007 ISSN 1676-0603.

O grimpeiro Leptasthenura setaria é totalmente dependente do pinheiro-do-paraná Araucaria angustifolia e considerado quase ameaçado de extinção pela IUCN. Apresentam-se sete novas localidades de ocorrência do grimpeiro para o Estado de São Paulo, incluindo os primeiros espécimes coletados dentro dos seus limites. A espécie foi encontrada tanto em pinheiros-do-paraná nativos quanto em plantados e está presente em cinco unidades de proteção integral.

Palavras-chave: Araucaria angustifolia, conservação, Floresta Ombrófila Mista, grimpeiro. 


\section{Introdução}

O grimpeiro, Leptasthenura setaria é uma espécie totalmente associada ao pinheiro-do-paraná Araucaria angustifolia (Bertol.) Kuntze. Alimenta-se de artrópodes encontrados nas folhas e galhos, fazendo seu ninho com ramos secos do próprio pinheiro, chamados popularmente de grimpas (Sick 1997). Ocorre desde a região serrana do Estado do Rio de Janeiro até o Rio Grande do Sul e em Misiones - Argentina (Sick 1997, Remsen 2003). É considerado quase ameaçado de extinção pela IUCN, devido à drástica redução da Floresta Ombrófila Mista (IUCN 2006).

Para o Estado de São Paulo há poucas localidades de ocorrência relatadas (Willis \& Oniki 2003): Campos do Jordão, Itapeva, Itapirapuã Paulista, Itararé, Piquete e Serra da Bocaina. Estes mesmos autores indicaram que não existem espécimes paulistas depositados nos museus por eles visitados. Bencke et al. (2006) registram a espécie para a Serra da Mantiqueira em São José dos Campos.

Relatamos aqui novas localidades de ocorrência para o grimpeiro no Estado de São Paulo, incluindo aí os primeiros espécimes coletados para o Estado. Além disso, o padrão de distribuição da espécie em São Paulo é discutido brevemente.

\section{Material e Métodos}

As localidades onde foram obtidos os registros são apresentadas na Tabela 1. Em campo foram utilizados binóculos Leica $10 \times 42$, Mirador 8 × 40 e Nikon $8 \times 32$, gravadores Nagra E com microfone Sennheiser MKH 816 T, TCM 5000 EV com microfone Sennheiser ME 66 e Marantz PMD670 com microfone Sennheiser K6/ME67, e GPS Garmin e Trex Summit.

\section{Resultados e Discussão}

Na Tabela 2 são apresentados os autores e a forma de documentação dos registros aqui divulgados. Considerando-se todos os dados disponíveis, evidencia-se a existência de pelo menos três grandes áreas de distribuição para a espécie no Estado (Figura 1): Sudoeste-Serra de Paranapiacaba, Serra da Mantiqueira e Serra da Bocaina-Serra do Mar Norte.

A presença do grimpeiro em pinheiros-do-paraná plantados, tem sido considerada como um indício da ocorrência natural, mesmo que pretérita, da árvore numa determinada região (Sick 1997). Cada local de ocorrência citado acima foi alocado em um mapa base com a distribuição da Floresta Ombrófila Mista (Floresta de Araucárias) e suas áreas de transição em São Paulo (Figura 2). Observa-se que algumas localidades não se encontram dentro da distribuição original dessa fitofisionomia, sugerindo que nessas áreas o grimpeiro utiliza pinheiros-do-paraná plantados. No caso do Parque Estadual Carlos Botelho, a vegetação original é a Floresta Ombrófila Densa e foram estabelecidos talhões de pinheiros-do-paraná, para pesquisa em produção de madeira, entre 1968 e 1970 (Ferraz \& Varjabedian 1999). Tais fatos indicam a capacidade do grimpeiro em colonizar novas áreas e a necessidade de pesquisas sobre a sua dispersão.

Quanto à conservação da espécie no Estado de São Paulo, os dados disponíveis demonstram que ela está presente em pelo menos cinco unidades de proteção integral: Estação Ecológica de Bananal, Parque Estadual de Campos do Jordão, Parque Estadual Carlos Botelho, Parque Estadual da Serra do Mar e Parque Nacional da Serra da Bocaina. Como se trata de uma espécie de pequeno porte e que não é alvo de caça ou captura, se conclui que as populações nessas unidades encontram-se relativamente seguras.

\section{Agradecimentos}

Agradecemos à Marina Mitsue Kanashiro da Seção de Inventário do Instituto Florestal, pela confecção da Figura 2, ao Tessio Novack pela confecção da Figura 1 e aos responsáveis pelas unidades de conservação visitadas. AZA agradece ao Klaus Barretto e à Mônica C. de Brito da Casa da Floresta Assessoria Ambiental, pela oportunidade de trabalhar na região de Capão Bonito, e à Natália Macedo Ivanauskas por informações sobre o pinheiro-do-paraná. Somos gratos a dois revisores anônimos pelas críticas e sugestões à primeira versão do trabalho.

Tabela 1. Localidades dos novos registros do grimpeiro Leptasthenura setaria para o Estado de São Paulo.

Table 1. New localities to Araucaria Tit-Spinetail Leptasthenura setaria in the state of São Paulo.

\begin{tabular}{|c|c|c|}
\hline Localidade & Coordenadas & Município \\
\hline Catuçaba & $\begin{array}{l}23^{\circ} 14^{\prime} \mathrm{S} \\
45^{\circ} 07^{\prime} \mathrm{W}\end{array}$ & São Luiz do Paraitinga \\
\hline São José dos Alpes & $\begin{array}{l}22^{\circ} 55^{\prime} \mathrm{S} \\
45^{\circ} 27^{\prime} \mathrm{W}\end{array}$ & Pindamonhangaba \\
\hline Pedra do Baú & $\begin{array}{l}22^{\circ} 41^{\prime} \mathrm{S} \\
45^{\circ} 41^{\prime} \mathrm{W}\end{array}$ & São Bento do Sapucaí \\
\hline Estação Ecológica de Bananal & $\begin{array}{l}22^{\circ} 48^{\prime} \mathrm{S} \\
44^{\circ} 22^{\prime} \mathrm{W}\end{array}$ & Bananal \\
\hline Parque Estadual da Serra do Mar - Núcleo Cunha & $\begin{array}{l}23^{\circ} 14^{\prime} 08^{\prime \prime} \mathrm{S} \\
45^{\circ} 01^{\prime} 14^{\prime \prime} \mathrm{W}\end{array}$ & Cunha \\
\hline Rodovia SP 258 & $\begin{array}{l}23^{\circ} 57^{\prime} 10^{\prime \prime} \mathrm{S} \\
48^{\circ} 26^{\prime} 26^{\prime \prime} \mathrm{W}\end{array}$ & Capão Bonito \\
\hline Parque Estadual Carlos Botelho - Núcleo São Miguel Arcanjo & $\begin{array}{l}24^{\circ} 03^{\prime} 38^{\prime \prime} \mathrm{S} \\
47^{\circ} 59^{\prime} 16^{\prime \prime} \mathrm{W}\end{array}$ & São Miguel Arcanjo \\
\hline
\end{tabular}


Tabela 2. Autores e forma de documentação dos novos registros do grimpeiro Leptasthenura setaria para o Estado de São Paulo. MHNT = Museu de História Natural de Taubaté.

Table 2. Authors and documentation of new records of Araucaria Tit-Spinetail Leptasthenura setaria from state of São Paulo. MHNT = Taubaté Natural History Museum.

\begin{tabular}{|c|c|c|c|}
\hline Localidade & Autor & Data & Documentação \\
\hline Catuçaba & H. Alvarenga & 09/1995 & $\begin{array}{l}\text { Peles de dois machos: MHNT }-3.231 \\
\text { e } 3.243 \text {. }\end{array}$ \\
\hline São José dos Alpes & H. Alvarenga & $02 / 1972$ & $\begin{array}{l}\text { Sem documentação (registros visuais e } \\
\text { sonoros) }\end{array}$ \\
\hline São Bento do Sapucaí & H. Alvarenga & $02 / 1972$ & $\begin{array}{l}\text { Sem documentação (registros visuais e } \\
\text { sonoros) }\end{array}$ \\
\hline Estação Ecológica de Bananal & L. F. Silveira & $12 / 2003$ & Gravação de vocalização \\
\hline $\begin{array}{l}\text { Parque Estadual da Serra do Mar - Núcleo } \\
\text { Cunha }\end{array}$ & A. S. R. dos Santos & $03 / 2004$ & Gravação de vocalização \\
\hline Rodovia SP 258 & A. Z. Antunes & $05 / 2005$ & $\begin{array}{l}\text { Sem documentação (registros visuais e } \\
\text { sonoros) }\end{array}$ \\
\hline $\begin{array}{l}\text { Parque Estadual Carlos Botelho - Núcleo São } \\
\text { Miguel Arcanjo }\end{array}$ & $\begin{array}{l}\text { A. Z. Antunes, M. R. de Eston e G. } \\
\text { V. Menezes }\end{array}$ & $05 / 2006$ & Gravação de vocalização \\
\hline
\end{tabular}

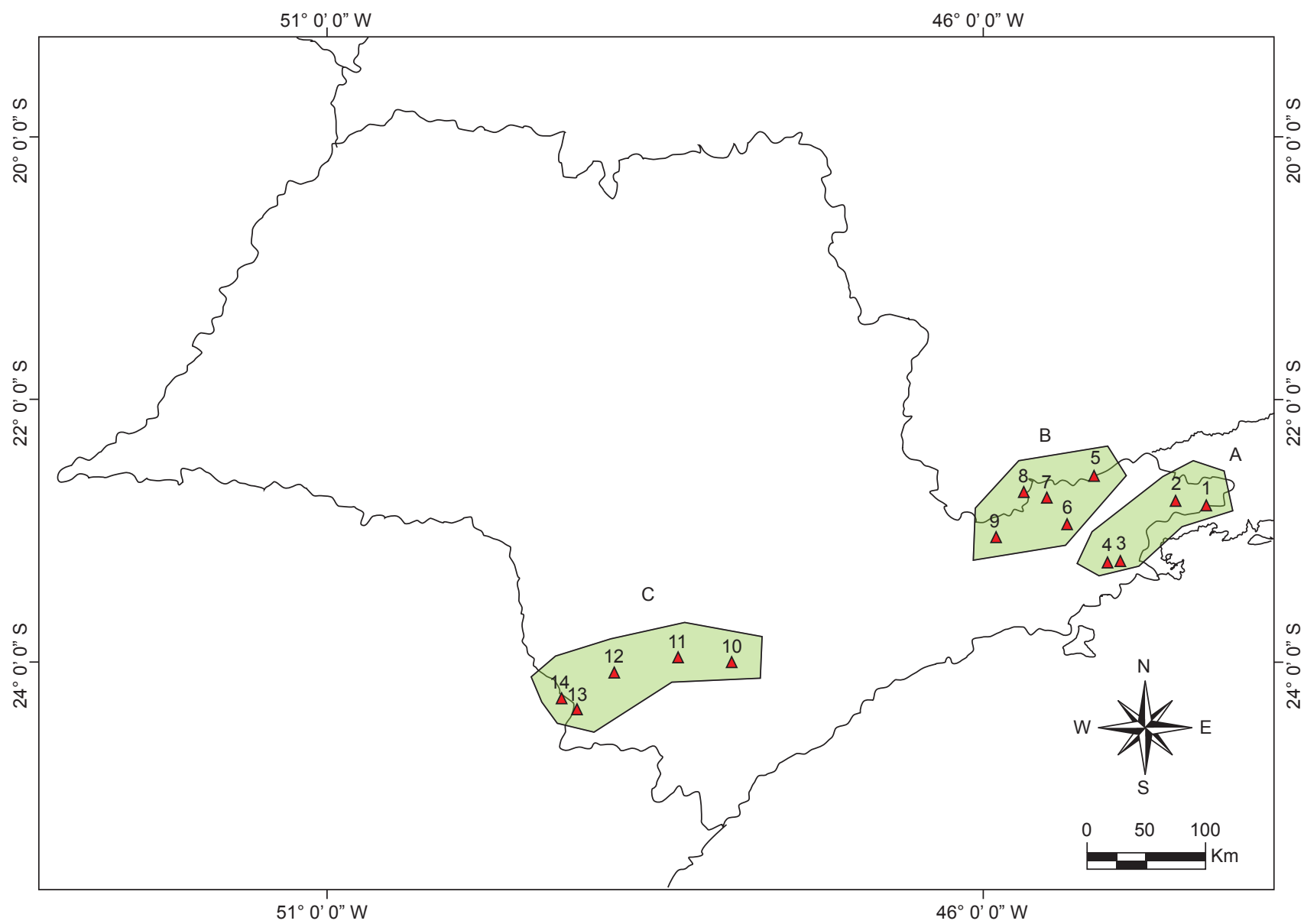

Figura 1. Distribuição do grimpeiro Leptasthenura setaria no Estado de São Paulo: 1. Bananal; 2. Serra da Bocaina; 3. Cunha; 4. Catuçaba - São Luiz do Paraitinga; 5. Piquete; 6. Pindamonhangaba; 7. Campos do Jordão; 8. São Bento do Sapucaí; 9. São José dos Campos; 10. Parque Estadual Carlos Botelho São Miguel Arcanjo; 11. Capão Bonito; 12. Itapeva; 13. Itapirapuã Paulista e 14. Itararé. Em verde, as três grandes áreas de ocorrência no estado: A. Serra da Bocaina-Serra do Mar; B. Serra da Mantiqueira; e C. Sudoeste-Serra de Paranapiacaba.

Figure 1. The records of Araucaria Tit-Spinetail Leptasthenura setaria in São Paulo: 1. Bananal; 2. Serra da Bocaina; 3. Cunha; 4. Catuçaba - São Luiz do Paraitinga; 5. Piquete; 6. Pindamonhangaba; 7. Campos do Jordão; 8. São Bento do Sapucaí; 9. Parque Estadual Carlos Botelho - São Miguel Arcanjo; 10. Capão Bonito; 11. Itapeva; 12. Itapirapuã Paulista e 13. Itararé. In green, the three principal Tit-Spinetail ranges in São Paulo: A. Serra da Bocaina-Serra do Mar; B. Serra da Mantiqueira; and C. Southwestern-Serra de Paranapiacaba. 


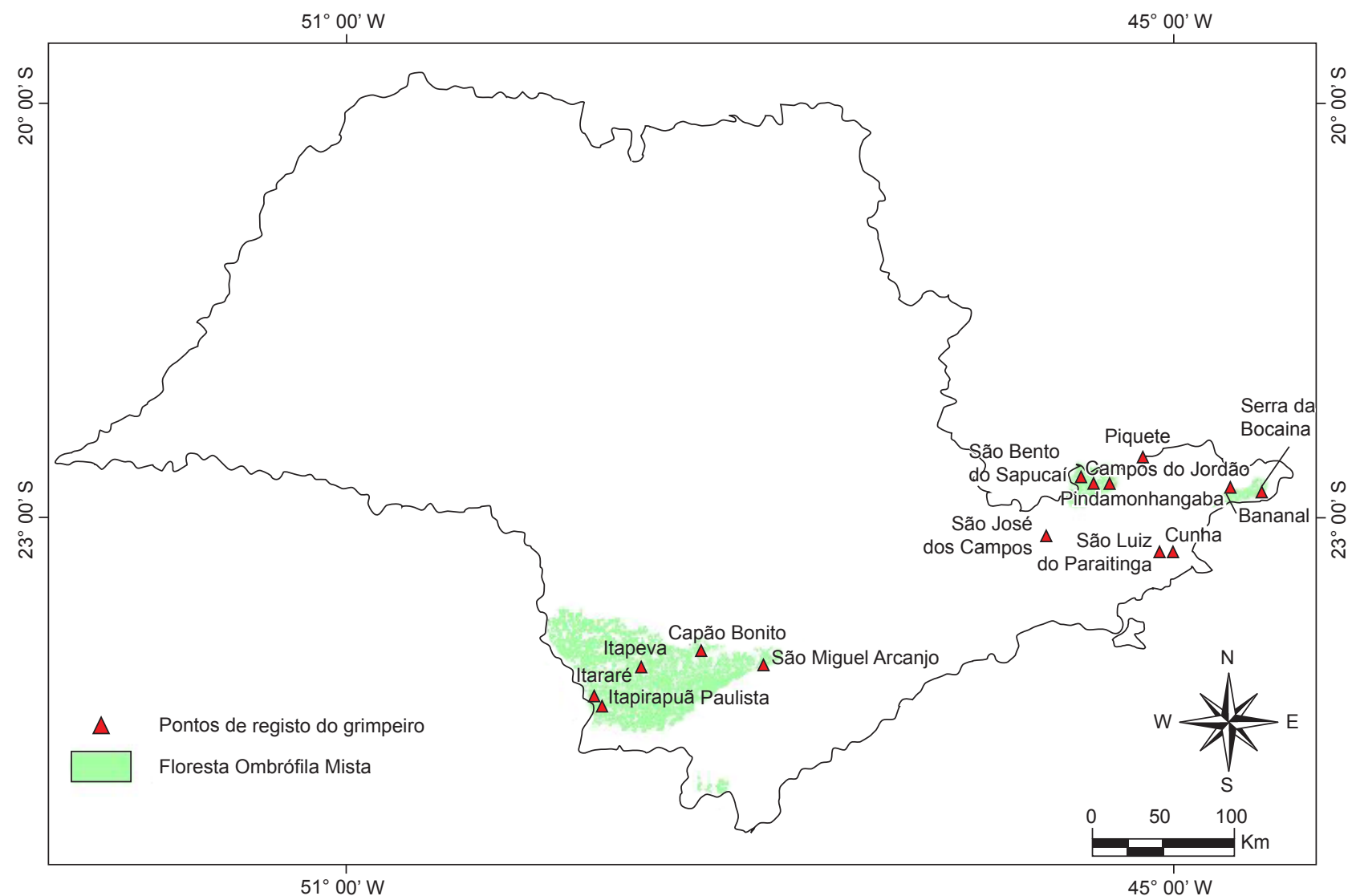

Figura 2. Área de ocorrência original da Floresta Ombrófila Mista no Estado de São Paulo e localidades em que o grimpeiro Leptasthenura setaria foi encontrado.

Figure 2. Original range of Araucaria Forest in São Paulo and Tit-Spinetail Leptasthenura setaria localities.

\section{Referências Bibliográficas}

BENCKE, G.A., MAURÍCIO, G.N., DEVELEY, P.F. \& GOERCK, J.M. (Org.). 2006. Áreas importantes para a conservação das aves no Brasil. Parte I - Estados do domínio da Mata Atlântica. SAVE Brasil, São Paulo.

FERRAZ, L.P.M. \& VARJABEDIAN, R. 1999. Evolução histórica da implantação e síntese das informações disponíveis sobre o Parque Estadual Carlos Botelho. Secretaria do Meio Ambiente/Instituto Florestal, São Paulo.

IUCN 2006. IUCN Red List of Threatened Species: http://www.iucnredlist. org (último acesso em 11/08/2006)

REMSEN, J.V., Jr. 2003. Family Furnariidae (Ovenbirds). In Handbook of the Birds of the World (J. del Hoyo, A. Elliott \& D. A. Christie, eds). Lynx Editions, Barcelona, vol. 8, p.162-357.

SICK, H. 1997. Ornitologia Brasileira: uma introdução. Editora Nova Fronteira, Rio de Janeiro.

WILLIS, E.O. \& ONIKI, Y. 2003. Aves do Estado de São Paulo. Editora Divisa, Rio Claro.
Titulo: Distribuição de Leptasthenura setaria

(Temminck, 1824) (Aves: Furnariidae) no Estado de São Paulo.

Autores: Antunes, AZ, Alvarenga, H, Silveira, LF, Eston, MR, Menezes, GV e Santos, ASR

Biota Neotropica, Vol.7 (número 1): 2007

http://www.biotaneotropica.org.br/v7n1/pt/abstract?shortcommunication+bn00607012007

Recebido em 04/10/06 - Versão reformulada recebida em 16/11/06 - Publicado em 01/01/07

ISSN 1676-0603 